\title{
CAUSAS E CONSEQÜÊNCIAS DO IMPACTO AMBIENTAL DA EXPLORAÇÃO DOS RECURSOS MINERAIS MARINHOS.
}

\author{
Abílio S. Gomes ${ }^{1}$, Jorge J. C. Palma ${ }^{2}$ \& Cleverson G. Silva ${ }^{2}$
}

Received August 24, 2000 / Accepted August 08, 2001

As atividades de mineração no mar podem causar diversos tipos de impactos ambientais aos ecossistemas marinhos, principalmente devido à destruição de habitats, que é um dos principais fatores que causam o declínio do número de espécies em todo o globo. Além de interferir diretamente no fundo submarino, as atividades de mineração podem causar um aumento da turbidez da água, com conseqüências para a produtividade primária local. Podem, introduzir e promover a liberação de nutrientes, causando a eutrofização e também a introdução de substâncias tóxicas, que quando incorporadas à biota, alteram o crescimento, a taxa de reprodução e a sobrevivência das espécies. Os métodos para identificação dos impactos ambientais das atividades de mineração no mar visam estabelecer se estas introduzem poluentes, determinar a biodisponibilidade desses poluentes, verificar a existência de respostas mensuráveis do ambiente e estabelecer a relação causal entre resposta e poluentes. Estes métodos empregam três abordagens: mensuração de concentrações de poluentes no meio físico (água e sedimento) e biótico (bioacumulação); estudos de laboratório ou de campo que visam estabelecer a existência de respostas toxicológicas dos organismos aos poluentes; e estudos de campo sobre modificações na estrutura e processos dos ecossistemas.

Palavras-chave: Ecossistemas marinhos, Impacto ambiental, Mineração marinha.

ENVIRONMENTAL IMPACTS FROM THE MARINE MINERAL EXPLORATION - The offshore extraction of marine minerals can cause several environmental impacts to the marine ecosystems, being the habitat destruction the main factor affecting the decline of the number of species around the world. Besides direct interference on the sea-bottom, the marine mineral activities can cause an increase in the water turbidity, affecting the local primary production. These activities can introduce and promote nutrient availability causing eutrophication. Otherwise it can introduce toxic substances that may be incorporated by the organisms, causing growth changes and alterations on the rates of reproduction and survival of the species. Current methods to identify the environmental impacts associated with the offshore mineral exploitation are centered on the reconnaissance of pollutant introduction and bio-availability, on the verification of measurable environmental changes and on the establishment of the relationship among the environmental response and pollutants. These methods apply three approaches: measurement of the pollutant concentration on the physical (water and sediment) and biological (bio-accumulation) environment; laboratory and field determinations of the organisms toxicity responses to pollutants; and field investigations of the ecosystem's structure and processes.

Key words: Marine ecosystems; Environmental impacts; Marine Mining.

\author{
${ }^{1}$ Universidade Federal Fluminense \\ Departamento de Biologia Marinha \\ Cx. Postal 100644, Niterói, RJ, 24.001-970 \\ Telefone e fax: (5521)2717-2041 \\ Email: abiliosg@vm.uff.br \\ ${ }^{2}$ Universidade Federal Fluminense \\ Departamento de Geologia/Laboratório de Geologia Marinha - LAGEMAR \\ Av. Litorânea s.n., Gragoatá, Niterói, RJ, 24.210-340 \\ Telefone e fax: (5521)2719-4241 \\ Email: jorge@igeo.uff.br / cleverson@igeo.uff.br
}




\section{PRINCIPAIS RECURSOS MINERAIS MARINHOS DO BRASIL}

Segundo Amaral et al. (1972), os minerais marinhos com potencial de exploração no Brasil são o petróleo, a sal-gema, o potássio, o enxofre, a gipsita, o carvão mineral, a ilmenita, o rutilo, o zircão, a monazita e os sedimentos fosfatados. Além disso, nódulos metálicos ocorrem em regiões profundas ao largo da margem continental e extensas reservas de areias terrígenas e sedimentos calcários, formados principalmente por algas, ocorrem desde a costa do Pará até o Espírito Santo.

De longe, o petróleo é o mineral marinho mais explorado atualmente no Brasil. De acordo dados da Agência Nacional de Petróleo (www.anp.gov.br) a produção de petróleo em campos submarinos representou quase $83 \%$ da produção nacional no ano de 2000. A maior parte das reservas de petróleo de origem marinha encontra-se na Bacia de Campos, situada na margem continental dos Estados do Rio de Janeiro e Espírito Santo, perfazendo 95\% da produção marinha brasileira.

\section{PRINCIPAIS ATIVIDADES MINERADORAS COM POTENCIAL POLUIDOR}

Assim como várias outras atividades humanas, as atividades de mineração podem causar diversos tipos de impactos ambientais aos ecossistemas marinhos, podendo prejudicar a pesca, afetar o turismo e as atividades recreativas, além de causar problemas de saúde pública.

As atividades de mineração, causadoras de impactos ao ambiente marinho, podem ser desenvolvidas tanto em regiões continentais próximas à costa, como no próprio oceano. Na mineração de áreas continentais, além da extração do mineral desejado, são produzidos rejeitos oriundos de túneis e galerias que são construídos para facilitar o acesso às jazidas. Esses rejeitos podem ser armazenados em áreas continentais, provocando modificações na paisagem costeira, ou, alternativamente, serem despejados no mar.

Dentre as atividades realizadas nos continentes, as minerações de carvão, cobre, molibdênio, chumbo, zinco, alumínio e ferro têm sido reportadas como causadoras de impacto em mares em vários locais do globo (Ellis, 1988).

As principais atividades mineradoras realizadas no ambiente marinho propriamente dito são a extração de areia e cascalho para utilização na construção civil, a explotação de algas calcárias e conchas, a mineração de nódulos polimetálicos em mar profundo e a exploração de hidrocarbonetos de petróleo.

No Brasil, com exceção do petróleo, a explotação de recursos minerais marinhos tem sido pontual e descontínua, restringindo-se à extração de areias para regeneração de praias e extração localizada de conchas e algas calcárias no Rio de Janeiro e Espírito Santo.

\section{MEDIDAS DE IMPACTO AMBIENTAL DAS ATIVIDADES DE MINERAÇÃO}

As técnicas utilizadas para medir os impactos causados pelas atividades de mineração não diferem das utilizadas para estudar impactos provocados por outras atividades antrópicas. Os métodos empregados visam estabelecer se as atividades mineradoras introduzem poluentes no ambiente, determinar a biodisponibilidade desses poluentes, verificar a existência de respostas mensuráveis do ambiente e estabelecer a relação causal entre resposta e poluentes.

As medidas de impacto podem ser realizadas nos diversos níveis de organização dos seres vivos: subcelular, celular, populacional e ecossistêmico. Impactos observados em níveis inferiores de organização podem ter implicações nos níveis superiores. Os efeitos observados a nível subcelular, celular e populacional refletem na estrutura das comunidades bióticas, podendo-se observar uma diminuição da biodiversidade e uma deterioração do patrimônio genético local. Entretanto, nem todo impacto observado a nível fisiológico, subcelular e celular irá refletir-se ao nível das populações. Deste modo, as análises da estrutura das comunidades e processos do ecossistema fornecem a palavra final sobre os impactos ambientais da mineração.

De acordo com Sinderman (1996) e outros autores, as respostas da biota aos poluentes são distribuídas no tempo; algumas ocorrendo 
imediatamente após o evento poluidor, enquanto que outras podem levar meses para se manifestar (Fig. 1). O diagrama apresentado na Fig. 2, proposto por Underwood e Peterson (1988), mostra os tipos de medidas realizados nos diferentes níveis de organização e as possíveis conexões entre esses níveis.

Existem três abordagens complementares que visam determinar a existência de impactos ambientais: a mensuração de concentrações de poluentes no meio físico (água e sedimento) e biótico (bioacumulação); os estudos de laboratório ou de campo que visam estabelecer a existência de respostas toxicológicas dos organismos aos poluentes; e os estudos de campo sobre modificações na estrutura e processos dos ecossistemas.

\section{IMPACTOS CAUSADOS ATIVIDADES MINERADORAS \\ PELAS}

As atividades de mineração causam tanto impactos diretos como indiretos no ambiente marinho. Essas atividades transformam o ambiente físico ou podem causar danos expressivos diretamente à fauna e flora. A ação dos instrumentos empregados nas atividades

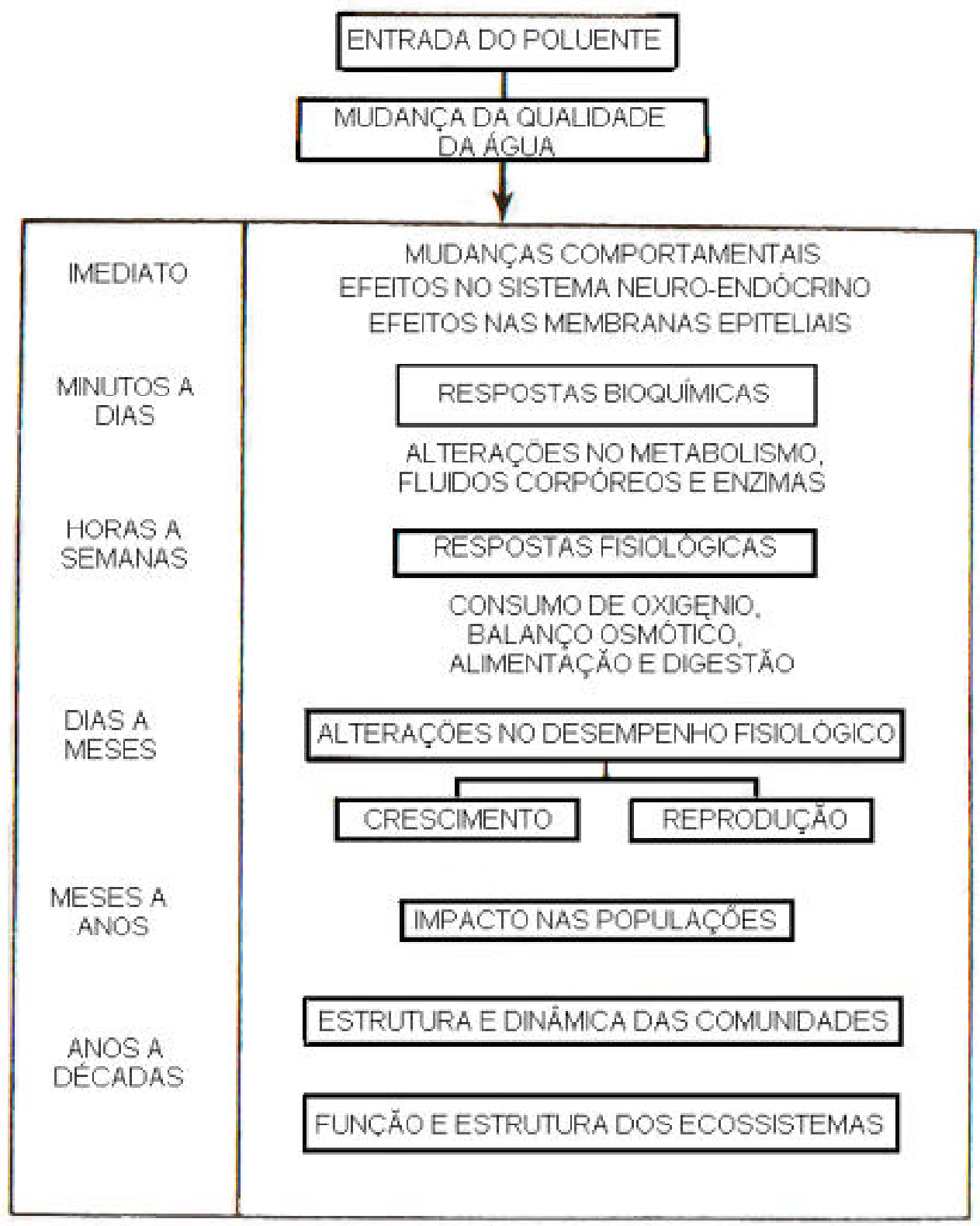

Figura 1 - Seqüência temporal dos efeitos do estresse de poluentes (modificado de Sinderman, 1996). 


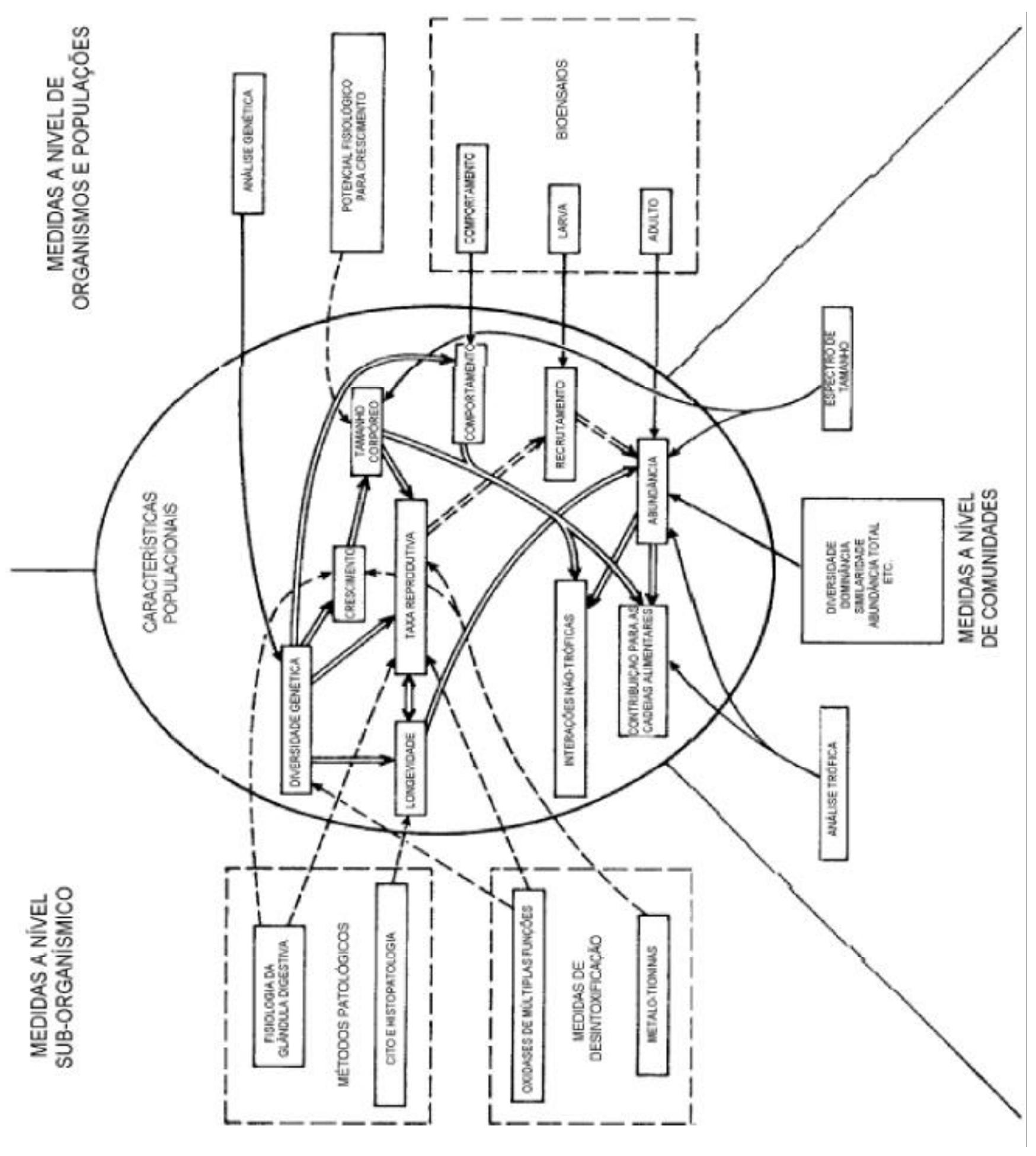

Figura 2 - Principais medidas de impacto de poluição realizadas nos diferentes níveis de organização dos seres vivos e suas conexões. As linhas contínuas indicam conexões já demonstradas para vários organismos marinhos. As linhas pontilhadas indicam conexões potenciais, porém ainda não demonstradas (Modificado de Underwood \& Peterson, 1988). 
mineradora pode causar a destruição ou uma grande transform ação doshabitats naturais.

A destruição de habitats é um dos principais fatores que causam o declínio do número de espécies em todo o globo. Segundo Stuart Pimm, da Universidade de Columbia, nos Estados Unidos, a destruição de habitats pode levar à perda de 50\% das espécies da Terra nos próximos 50 anos (Sih, Jonhsson \& Luikart, 2000). Além disso, durante a atividade de mineração observa-se, freqüentemente, a fuga de espécies com maior poder de locomoção e a morte por esmagamento de espécies sésseis e sedentárias.

A capacidade de modificação de habitats causada pela mineração pode ser muito expressiva. Segundo medidas conservadoras realizadas por Groot (1986), entre 1979 e 1984 foram extraídos 40 milhões de metros cúbicos de areia, 9 milhões de metros cúbicos de cascalho e 1 milhão de metros cúbicos de calcário dos mares europeus e da América do Norte. A movimentação de sedimentos para manutenção de canais de navegação e portos somou 400 milhões de metros cúbicos nesse mesmo período. A explotação de algas calcárias, por sua vez, totalizou $650 \mathrm{mil}$ metros cúbicos na França, em 1974.

Adicionalmente, a mineração marinha pode causar um aumento da turbidez da água, com conseqüências para a produtividade primária local e atividade de alimentação dos animais bentônicos. Pode, ainda, introduzir e promover a liberação de substâncias tóxicas que podem ser incorporadas à biota, num fenômeno de bioacumulação, ou serem passadas através das redes tróficas e apresentar concentrações elevadas nos organismos de topo de cadeia, num processo de biomagnificação biológica. Essas substâncias tóxicas, uma vez incorporadas pela biota, podem promover alterações fisiológicas, bioquímicas, genéticas e comportamentais, que por sua vez podem alterar o crescimento, a taxa de reprodução e a sobrevivência das espécies.

As atividades mineradoras podem, também, provocar a liberação de nutrientes para a coluna d'água. Esse aumento na concentração de nutrientes pode elevar a produtividade primária local, nem sempre com efeitos positivos para a biota, causando eutrofização das águas.
O despejo de rejeitos no mar ocorre tanto em áreas rasas, como em áreas profundas. $\mathrm{O}$ despejo de rejeitos em regiões profundas é realizado em várias partes do globo, e.g. no Canadá, Filipinas e Papua Nova Guiné. Geralmente é feito através de tubulações, com o material deslocando-se como uma corrente de turbidez, que caracteristicamente apresenta baixa dispersão no ambiente. Essas correntes de turbidez promovem um soterramento dos organismos bentônicos, sem, entretanto, causar declínio à pesca local, que depende das cadeias alimentares de águas rasas (Clark, 1997).

As regiões profundas também podem sofrer impactos devido à mineração de nódulos polimetálicos e crostas cobaltíferas. A mineração desses nódulos remove junto a infauna e a epifauna que colonizam esses nódulos. De acordo com Bluhm (1994) e Bluhm et al. (1995), a remoção de nódulos de manganês causa a destruição do habitat da fauna de fundos consolidados, resultando no desenvolvimento de uma fauna típica de fundos não consolidados, de baixa diversidade específica. Essas atividades de mineração produzem uma pluma de sedimentos que está sujeita à deriva pela ação das correntes de fundo, causando impactos em áreas que não sofreram distúrbios primários.

Também, a mineração de sulfetos hidrotermais em cordilheiras mesoceânicas, uma atividade cujo início é previsto para as próximas décadas, pode causar impactos a uma biota de características ímpares, pouco conhecida (Mello e Quental, neste volume). Os ecossistemas de oceano profundo são os que apresentam a maior biodiversidade do planeta, o que pode representar recursos cujo valor ainda não se pode avaliar.

Ellis (1988) identificou quatro tipos de impactos primários causados pelos despejos de uma mina de cobre no Canadá: aumento da turbidez da água, soterramento do leito submarino, bioacumulação de metais traços e destruição de habitats costeiros. Segundo este autor, os três primeiros impactos ocorrem sistematicamente devido à mineração marinha. $\mathrm{O}$ aumento da turbidez da água pode potencialmente reduzir a produtividade primária biológica e, como conseqüência, pode reduzir a produção pesqueira. O soterramento do leito 
submarino pode causar mortalidade em espécies pesqueiras de peixes e invertebrados bentônicos.

As atividades de mineração de hidrocarbonetos de petróleo (gás e óleo), tanto em suas etapas de exploração quanto de produção, são potenciais causadoras de impacto ambiental. As atividades de pesquisas sísmicas, perfuração dos poços, produção e transporte desses compostos apresentam riscos à biota marinha. As ondas sísmicas utilizadas na prospecção das reservas petrolíferas podem interferir no sistema de ecolocação de mamíferos marinhos e ser letal para espécies de peixes. A perfuração de poços promove um aumento da turbidez da água, $\mathrm{o}$ soterramento do leito submarino e a contaminação química da água e sedimentos. As plataformas de produção e os meios de transporte representam fontes constantes de derrames acidentais e operacionais, podendo causar contaminação da biota. Ao redor das plataformas de produção observa-se um gradiente de contaminação química e da concentração de matéria orgânica, observando-se uma sucessão de espécies e variações na abundância e diversidade biológica.

A natureza do impacto e as conseqüências para o ambiente das atividades offshore da indústria de petróleo podem ser resumidos conforme a Tab. 1, modificada de Patin (1999).

No Brasil, devido à magnitude das reservas e o estado atual de exploração das mesmas, a mineração de petróleo e gás natural é a que representa maior potencial de impacto para o ambiente marinho.

A produção de petróleo e gás ao largo da costa do Brasil está restrita a poucos estados (Rio Grande do Norte, Sergipe, Bahia, Espírito Santo, Rio de Janeiro e São Paulo), estando a maior parte da exploração e das atividades da indústria petroquímica concentrada na região sudeste. Além das atividades de exploração, dutos e navios utilizados para o transporte e terminais petrolíferos apresentam riscos constantes de derrame de petróleo no mar.

Recentemente Silva et al. (1997) avaliaram o impacto da poluição por petróleo na costa brasileira utilizando dados compilados da literatura. Segundo os dados apresentados, os valores de concentração de óleo em sedimentos e de bioconcentração em peixes e moluscos indicam uma contaminação entre moderada e baixa, com exceção de amostras coletadas em eventos de poluição aguda. Igualmente, os costões rochosos, muito comuns na região sudeste, parecem terem sido pouco afetados pelos constantes derrames. Os manguezais, por sua vez, parecem sofrer bastante com a poluição por petróleo. Quando atingidos por derrames de óleo apresentam perda substancial de folhas, aumento no número de raízes aéreas, malformações de folhas e frutos, além da diminuição da produção de serrapilheira. Os autores enfatizam que os estudos sobre os efeitos da poluição por petróleo ainda são muito escassos no Brasil e recomendam pesquisas de longa duração para detectar mudanças na estrutura das comunidades e efeitos subletais que possam estar ocorrendo nas populações de locais mais sujeitos à exposição ao petróleo.

A identificação dos efeitos adversos das atividades relacionadas à produção, transporte e processamento

\begin{tabular}{|c|c|c|c|c|}
\hline $\begin{array}{c}\text { NATUREZA DO IMPACTO E SUAS } \\
\text { CONSE QQUNNCIAS }\end{array}$ & $\begin{array}{c}\text { EXPLORAÇAO E } \\
\text { PESQUUISAS } \\
\text { SI SMICAS }\end{array}$ & $\begin{array}{l}\text { COLOCACAO DE } \\
\text { ESTRUTURAS }\end{array}$ & $\begin{array}{l}\text { PERFURAÇAO E } \\
\text { ATIVIDADES DE } \\
\text { EXPL ORAÇÃO }\end{array}$ & TRANSP ORTE \\
\hline \multicolumn{5}{|l|}{-IMP ACTO FÍSICO SOBRE: } \\
\hline Espécies Pelágicas & + & + & - & + \\
\hline$\overline{\text { Espécies B entûnicas }}$ & + & + & + & + \\
\hline Biótopos Bentônicos & + & + & + & + \\
\hline -INTERFERÊNCIA NA PESCA & + & + & + & + \\
\hline $\begin{array}{l}\text { •DISTÚRDIO GODRE } \\
\text { ESPÉCIES MIGRATÓRIAS }\end{array}$ & + & + & - & $t$ \\
\hline •POLUIÇÃO QUÍMICA & - & - & + & + \\
\hline -AUMENTO DA TURBIDEZ & + & + & + & - \\
\hline $\begin{array}{l}\text { •ODORIZAÇÃO OU } \\
\text { CONTAMINAÇÄO DE } \\
\text { ESPÉCIES COMERCIAIS }\end{array}$ & - & - & + & + \\
\hline
\end{tabular}

Nota: + e - significam, respectivamente, presença ou ausência de impacto e suas conseqüências durante as diferentes atividades.

Tabela 1 - Características gerais do impacto da indústria offshore de óleo e gás sobre os ecossistemas e biorecursos aquáticos (modificado de Patin, 1999). 
de óleo no Brasil tem se baseado muito em testes de toxicidade realizados com macroalgas, crustáceos copépodas, crustáceos misidáceos, camarões e moluscos. (Nipper, 2000). Esses estudos têm demonstrado efeitos negativos de efluentes de refinarias e água de produção das plataformas.

\section{CONCLUSÕES}

As atividades de mineração marinha, embora potencialmente e comprovadamente poluidoras, ampliam-se cada vez mais nos países desenvolvidos e mesmo naqueles em desenvolvimento fornecendo hoje razoável parcela de recursos minerais e energéticos para os estados costeiros. Com a descoberta de novos recursos potenciais em águas cada vez mais profundas e com o esgotamento das reservas em terra, é inevitável o incremento destas atividades para suprir as demandas de uma população mundial igualmente crescente.

É, portanto, imperativo que os empreendimentos atuais e novas investidas exploratórias e explotatórias de recursos minerais marinhos, sejam feitas com base em estudos prévios de detalhe sobre o meio-ambiente, acompanhadas de campanhas de monitoramento que visem quantificar a capacidade de recolonização dos ambientes afetados. Além disso, devem ser adotados protocolos rigorosos para escolha de métodos de exploração e explotação e avaliação de impacto, incluindo testes de operação de sistemas de lavra, além de garantir a implementação de medidas para mitigação do impacto ambiental.

No caso das atividades de extração dos sulfetos e sedimentos metalíferos, em áreas de atividade hidrotermal intensa, deve-se, antes de tudo, proceder a uma avaliação realista da relação custo/benefício da atividade extrativa mineral, tendo em vista o alto valor potencial em termos de desenvolvimento biotecnológico, de biotas ainda muito pouco conhecidas.

\section{REFERÊNCIAS}

AMARAL， C.A.B.; VICALVI, M.A.; BARRETO, L.A. \& SANTANA, C.I., 1972. Recursos minerais da margem continental brasileira. Anais do XXVI Congresso Brasileiro de Geologia, 2: 289-299.

ASMUS, H.E. \& GUAZELLI, W., 1981. Descrição sumária das estruturas da margem continental brasileira e das áreas oceânicas e continentais adjacentes - hipóteses sobre o tectonismo causador e implicações para o prognóstico do potencial de recursos minerais. Série Projeto RMAC, 9: 187-269.

BLUHM, H., 1994. Monitoring megabenthic communities in abyssal manganese nodule sites of the East Pacific Ocean in association with commercial deep-sea mining. Aquatic Conservation: Marine and Freshwater Ecosystems, 4: 187-201.

BLUHM, H.; SCHRIEVER, G \& THIEL, H., 1995. Megabenthic recolonization in an experimentaly disturbed abyssal manganese nodule area. Marine Georesources and Geotehnology, 13: 393-416.

CLARK, R.B., 1997. Marine pollution. Clarendon Press, $4 \underline{\text { th }}$ ed., Oxford, $161 \mathrm{p}$.

ELLIS, D.V., 1988. Case history of coastal and marine mines. In: Salomons, W. \& Forstner, U.(eds.). Environmental management of solid waste. Springer-Verlag, Berlin, 396 p.

ELLIS, D.V. \& TAYLOR,L.A., 1988. Biological engineering of marine tailings beds. In: Salomons, W. \& Förstner, U. (eds.). Environmental management of solid waste. Springer-Verlag, Berlin, $396 \mathrm{p}$.

ELLIS, D.V. \& HOOVER, P.M., 1990. Benthos recolonizing mine tailings in British Columbia fjords. Marine Mining, 9: 441-457.

GROOT, S.J., 1986. Marine sand and gravel extraction in the North Atlantic and its potential environmental impact, with emphasis on the North Sea. Ocean Management, 10: 21-36.

MELLO, S. L. M. \& QUENTAL, S.H.A.J, Depósitos de Sulfetos Metálicos no Fundo dos Oceanos, neste volume.

NIPPER, M., 2000. Current approaches and future directions for contaminant-related impact assessments in coastal environments: Brazilian perspective. Aquatic Ecosystem Health and Management, 3: 433-447. 
PATIN, S., 1999. Environmental impact of the offshore oil and gas industry. EcoMonitor, New York, $425 \mathrm{p}$.

SIH, A.; JONHSSON, B.G. \& LUIKART, G., 2000. Habitat loss: ecological, evolutionary and genetic consequences. Trends in Ecology and Evolution, 15(4): 132-134.

SILVA, E.M.; PESO-AGUIAR， M.C.; NAVARRO, M.F.T.\& CHASTINET, C.B.A., 1997. Impact of petroleum pollution on aquatic coastal ecosystem in Brazil. Environmental Toxicology and Chemistry, 16(1): 112-118.

SINDERMAN, C.J., 1996. Ocean pollution. Effects on living resources and humans. CRC Press, Boca Raton, 275 p.

UNDERWOOD, A.J. \& PETERSON, C.H., 1988. Towards an ecological framework for investigating pollution. Marine Ecology Progress Series, 46: 227-234.

www.anp.gov.br - Web page da Agência Nacional de Petróleo - ANP.

\section{NOTE ABOUT THE AUTHORS}

\begin{abstract}
Abílio S. Gomes
Biólogo formado pela Faculdade Maria Teresa em 1984, mestre pelo Museu Nacional da Universidade Federal do Rio de Janeiro (1989) e doutor pelo Instituto Oceanográfico da Universidade de São Paulo (1997). Foi professor da Universidade Federal do Espírito Santo em 1992 e atualmente é professor adjunto da UFF, com atuação no Departamento de Biologia Marinha, ministrando as disciplinas de Ecologia Marinha e Ecologia de Sedimentos junto ao programa de mestrado em Biologia Marinha. Seu interesse depesquisa envolve o estudo de ecologia dos bentos marinhos, com aplicações no monitoramento e controle de poluição, principalmente associada ao impacto da poluição por petróleo.
\end{abstract}

\section{Jorge J. C. Palma}

Received his B.Sc. in Geology in 1967 from the Universidade Federal do Rio Grande do Sul (Brazil), M.Sc. in Geology in 1989 from the Universidade Federal do Rio de Janeiro (Brazil), and D.Sc. in Geophysics in 1998 from the Universidade de São Paulo. From 1967 to 2000, he worked for the Departamento Nacional da Produção Mineral and since 1984 he is a lecturer/ researcher at the Laboratório de Geologia Marinha of the Universidade Federal Fluminense (Brazil). His main research interests are tectonic evolution and crustal structure of oceanic fracture zones and continental margins, mid-ocean ridges, marine geological mapping, and marine mineral resources, and geological and geophysical aspects of the U. N. Convention on the Law of the Sea. He participated in numerous cruises of Brazilian and other country ships as well as in several working groups for the Brazilian Government.

\section{Cleverson G. Silva \\ Geólogo formado pela Universidade Federal do Rio de Janeiro (UFRJ) em 1982. Em 1987, obteve o grau de Mestre em Geologia pela UFRJ e, em 1991, o de doutor em Geologia Marinha pela Universidade de Duke, Carolina do Norte, EUA. Atualmente é professor e coordenador de Pós-Graduação do Depto. de Geologia/ LAGEMAR-UFF ministrando na pós-graduação as disciplinas de Geologia do Petróleo e Seminários de Campo em Geologia Sedimentar. Participou e coordenou diversas expedições oceanográficas no Brasil e no exterior voltadas ao estudo sobre sedimentação e estrutura da plataforma continental. Seu interesse de pesquisa envolve processos geológicos costeiros, recursos minerais marinhos e processos sedimentares em plataforma e talude.}

\section{ON/MCT - National Observatory}

Offers Graduate courses in Geophysics leading to Master's and PhD's degrees with emphasis in two major research topics: Solid Earth Geophysics and Exploration Geophysics. The program maintains regular scientific collaboration with several universities and research institutes in USA, England, Germany, Italy, Switzerland and others. The ON has a staff of 11

$\mathrm{PhD}$ and 4 collaborators. Browse http://obsn.on.br 